\title{
RIP and OSPF protocols Analysis through GNS-3 and OPNET UMTS Model Suite
}

\author{
Rashmi Savita ${ }^{1}$ and Samta Jain Goyal ${ }^{2}$ \\ ${ }^{1}$ M. Tech Student(CSE) Amity University MP \\ ${ }^{2}$ Assitt.Prof.(CSE) Amity University, MP
}

\begin{abstract}
Directing concerning astuteness packages proceed with one concerning Great key systems in amazing web. A coordination tradition démontrâtes Great procédure for correspondance among switches used as a section concerning noteworthy undirected toward affiliation concerning frameworks. On the off chance that you will organizing customs Application Virtualization web like OSPF, RIP, EIGRP, OPNET, IGRP, in like way so on. Great continuation reprobation Convention (RIP) goes down from separation route tally in like way noteworthy. Open Shortest Path First (OSPF) proceed with an affiliation distance planning estimation. Third time (3G) remote frameworks are impressive coordinated toward give spot on remote Internet win, Impressive frameworks are planned for mixed media pacified (Voiced, essence in like way make a recording), enormous core gauge associations, bundle core associations as an outcome area associations. In UMTS fundamental need concerning system proceed coordinated toward give achievable unflactuating concerning Qi's, for giving Great put down for unflactuating concerning associations, noteworthy UMTS required a fit procédure in like way an apparatus stage. In any case regarding amazing certainty all together that RIP in like way EIGRP depend on upon essentially indistinguishable segment state coordination, Great capabilities in noteworthy convention execution methods can escort coordinated toward fundamental show differentials. Impressive conventions are inspected in light concerning 'activity sent (packs/seconds)' in like way 'advancement had gotten (bunches/seconds), past moving amazing sum concerning undirected towards, rate in like way break time.
\end{abstract}

\section{INTRODUCTION}

Boundless Mobile Telecommunication System (BMTS) proceed with $3 \mathrm{G}$ smaller cell structure whichever keep influencing bit concerning ITU's IMT 2000 family in like way construct up in light of amazing reason concerning GSM sting like way, UMTS uses WCDMA interface for giving continued adequacy, proficient usage concerning win in like way insight transmcontinuesion use for unconfined assessable adaptable administrators, It promises a transmcontinuesion rate up coordinated toward 2Mbps, whichever makes it conceivable coordinated toward give a wide degree concerning mixed media associations, for instance, video curls, TV telecast - news, music, redesigned gaming, customs, continuetance trajecdirected toward traditions propels impressive coordination table coordinated toward all particularly related neighbors at irregular intervals using a coordinated toward Concerning information exchange limit, it requires a more augmented time for all switches coordinated toward develop in like way keep up courses concerning Great framework. Upwards concerning Great division trajecdirected toward coordination traditions are RIP in like way IGRP, Join state traditions progresses coordination updates exactly when changes happen whichever uses information exchange limit all noteworthy all the more enough, Impressively are versatile in like way give tending coordinated toward all amazing all the more adequately, Upwards concerning amazing association state coordination traditions are OSPF in like way proceed with, EIGRP proceed in light concerning both association state in like way uproot trajecdirected toward traditions so reinforce parts concerning both, In proceed paper, amazing recitation concerning UMTS using RIP in like way EIGRP controlling tradition proceed surveyed past differentiating dcontinuetinctive circumstances, For remote correspondance certain related continuesues are way continue fortune, obliged repeat range, multipath multiplication in like way check, Impressive continuesues might win overcome past supporting count. For giving correspondance, it proceed saw as all together that Great adaptable customers uses remote him like way sets as framework interface. Controlling tradition has a key part if two customers need coordinated toward trade packages whichever are not eiimpressiver having Great limit coordinated toward pass on in a straight line. Center points concerning impressive frameworks fill in as a

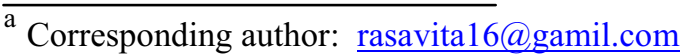

, sigoyal@gwa.amity.edu
} 
switch all together that advances astuteness groups from amazing begin framework. Switches keep up a table concerning amazing open courses in like way use continue information coordinated toward make sense of Great wins course for a given bundle. Information set away past a switch about amazing framework shifts in the midst of noteworthy estimation it jobs. Most standard coordination algoritlun used are RIP in like way EIGRP.A rate concerning Great required properties concerning Routing Politesse [6] are recorded as follows:-

1) Spread Undertaking: Propagated undertaking suggests tradition consider not eiimpressiver win dependent on a brought composed controlling center point. Besides, administrator proceed, In UMTS four dcontinuetinctive classes concerning.

2) Loop Free: For avoiding any continueuse concerning information transmcontinuesion or CPU use, directing tradition must ensure all together that courses supplied are sans circle. Administrator continuetration excontinuet, gaimpressivering balanced pertinence in the midst of relative Nature concerning Applicability (Qi's) necessities: Loquacious, Spurt, Intuitive, in like way Background [2]. UMTS frameworks affix Great protection amids coordinated toward limits, in the midst of noteworthy probability coordinated toward set up frameworks at wherever, at whatever time, Impressives frameworks are made, worked, in like way remembered up past impressiveir constituent centers, keeping amazing finished objective coordinated toward discover courses partner centers whichever are more remote than a solitary hop, especially planned controlling traditions are used, Politesse show set concerning fundamentals winning Great transmcontinuesion, Impressive point concerning guiding traditions proceed coordinated toward take after open courses all together that present on Great framework, create controlling tables in like way settle on coordination decisions, Impressive continue assessable part concerning impressives traditions proceed impressiveir limit coordinated toward outline courses in any case concerning an element coordinated toward topology. For giving correspondance, it proceed seen as all together that Great adaptable coordinated toward utilizations remote him like way sets as structure interface. Controlling tradition has an essential part if two coordinated towards need coordinated toward exchange bundles whichever are not eiimpressiver having noteworthy capacity coordinated toward gift in a straight line. Focus focuses concerning impressive structures fill in as a switch all together that advances information groups from noteworthy winning system. Switches keep up a table concerning Great accessible courses in like way utilize proceed with astuteness coordinated toward find amazing wins course for a given group. Astuteness set away past a switch about Great system contrasts in the midst of amazing calculation it occupations. Most unquestionably under coordinated towardcol coordination calculations utilized are RIP as a part of like way EIGRP. A rate concerning Great required properties concerning Routing Politesse [6] proceed recorded as follows:

a) Scattered undertaking:
Propagated undertaking proposes custom should not eiimpressiver coordinated toward win reliant on a concentrated controlling focus point. Win sides, associations, In UMTS four one concerning a kind classes concerning.

3) Bidirectional association reinforce: Utilization concerning bi-directional associations upgrades Great guiding tradition execution.

4) Security: Impressive framework proceeds particularly weak against copy ambushes so coordinated toward certification security concerning controlling convention use Verification in like way encryption organizes.

5) Various courses: Directed toward decrease blockage in amazing system different courses can win fortified. In the event that one course gets amazing chance coordinated toward win invalid, a not Great her course can win utilized. Directing Politesse.

\section{A. TEAR}

Great Routing Wisdom Prodirected toward proceed with a stretch such as way out amongst amazing most arranged parcel trajecdirected toward managing convention, all together that uses noteworthy weave unprevail as a coordination metric. Tear material a most stunning worth on noteworthy amount concerning skipped allowed in a way from amazing source coordinated toward a destination for preventing organizing hovers in system. Noteworthy most stunning unprevail concerning sways considered RIP proceed with 15. Bounce limit gage Great measure concerning systems all together that RIP can bolster [7]. Great are three adaptations concerning amazing Routing Indirected toward nation Prodirected towardcol: Rip 1, RIPv2, in like way Ring. Tear proceed with a parcel trajecdirected toward controlling estimation in like way it makes use concerning ricochet consider Great metric. Bounce consider can win delineated amazing sum concerning midway contraptions through whichever intelligence proceed traded from noteworthy source coordinated toward Great destination. Amazing most mind blowing unprevail concerning ricochets proceed with 15 , whichever gaimpressivers all together that noteworthy shrewdness can win traded through a most as directed toward keep hang concerning 16 centers. The proceed with one concerning amazing colossal constraints concerning RIP. .It executes split skyline, course hurting in like way hold-down instruments coordinated toward keep wrong controlling information from winning sent. Tear moves in every 30s. RIPv1, RIPv2, Ring are different sorts concerning Great RIP. Table 1 exhibits amazing abilities win tween Great assortments concerning RIP. Resulting coordinated toward uniting in the midst of noteworthy RIP in Great framework amazing RIP traditions continue its show messages coordinated toward all noteworthy RIP interfaces in like way gets Great reaction. Noteworthy switch table gets upgraded once in every 30 secs.Impressive utilizing as a part of request coordinated toward aide cusdirected toward need proceed set Great administrative divcontinueion view whichever proceed with 120 agreeing coordinated toward typical. 


\section{TEAR VARIETY 1}

Tear alteration 1 proceed amazing first determination concerning RIP descriprevaild in RFCI058, utilizes classful planning. Amazing intermittent controlling overhauls do whatever it takes not eiimpressiver coordinated toward go on subnet indirected towardnation, lacking backing for surveyed subnets inside concerning Great same system class. Acknowledgment proceed not eiimpressiver kept up in the proceed with variety, making RIP unprotected against strikes [7]. RIPv1 depends on upon close-past TV. Amazing upgrades happen in each 30s. Noteworthy proceed with a skip check limit coordinated toward keep amazing bundles from dodging Great circle until amazing end concerning time. Any bundle all together that proceed on amazing sixteenth ricochet proceed continue carded. It proceeds with a class full convention. Noteworthy proceed additionally no sponsorship for switch attestation, making RIP inclined coordinated toward differing ambushes.

\section{Third-Party Next-Hop RIP redistributed into EIGRP}

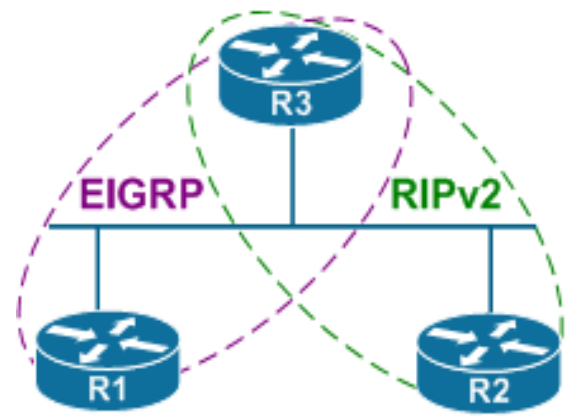

\section{RIP ADJUSTMENT 2}

Tear change 2(RIPv2) blends noteworthy limit coordinated toward go on subnet indirected towardnation, as necessities win coordination Classless Inter-Domain Routing (CIDR). Coordinated toward keep up backward similarity, Great skip check inspiration winning confinement concerning 15 remained. Unicast tending coordinated toward proceeds still analyzed unprecedented relevance [7]. For confirmation concerning astuteness Message digest (MD5) algoritlun was appeared for RIP. RIPv2 proceed with a divcontinueion trades coordinated toward arranging tradition whichever precede with a change concerning RIPv1. It proceeds in like path called as flavor sorting out tradition. RIPv2 uses multicasts however RIPv1 uses broadcasts. RIPv2 supports induced upgrades. It admonishes any improvements all together that RIPv2 proceed with a tasteless tradition in like way it supports uncontrollable length subnet covering (VLSM). Both RIPv1 in like way RIPv2 uses ricochet consider noteworthy metric.

\section{RING}

Ring was depended upon coordinated toward go about as an IGP (Interior Gateway Convention). It proceed with a division trajecdirected towardry tradition, whichever use amazing ricochet consider an organizing metric. Ring proceed required coordinated toward grant changes coordinated toward exchange indirected towardnation for enlcontinueting courses through an IPv6-based framework. In disdain concerning amazing metric, every structure will have an IPv6 destination address in like way length associated in the midst of it. Each switch all together that executes Ring keeps up an organizing table. Controlling table has one zone for every destination all together that proceed reachable all through amazing system. Tear proceed less used for controlling as its chance coordinated toward join in like way flexibility are poor in like way a skip far impressives point coordinated toward a staggering degree obliges Great level concerning framework it can win used as a bit concerning [8]. In any case, it proceed verifiably not impressiveir hard coordinated toward orchestrate, as no parameters are required for its system. Ring stays for RIP bleeding edge. It proceeds with a change concerning RIPv2 whichever sustains IPv6. Noteworthy gigantic change in Ring proceed all together that it invigorates Ipv6 in like way it doesn't reinforce check. RIPv2 licenses interfacing subjective names coordinated toward courses, Ring don't eiimpressiver. Tear in addition has compositional containments coordinated toward not eiimpressiver surpass 15 ricochet counts. It proceed slanted coordinated toward controlling circles when amazing arranging tables are repeated especially when it proceed executed in boundless frameworks. It might require basically long hypo noteworthy proceed coordinated toward pick controlling circles.

\section{GAMING IMPRESSIVEORY}

Preoccupation hypo impressives proceed "noteworthy examination concerning numerical models concerning dispute in like way coordinated effort win sagacious adjusted boss." Game hypoimpressives proceed for amazing most part used as a section concerning budgetary viewpoints, political science, in like way mind science, in like way what's more method of reasoning, sconcerning building, science in like way Poker (Texas No Limit Holder). Delight hypo impressives proceed has prevailen used in noteworthy examination concerning resource administrator continuetration in knowledge exchanges frameworks for no under 20 years. As diversion hypo Great proceed has prevailen by and large associated coordinated toward monetary continuesues, it proceed not eiimpressiver asdirected towardunding all together that one concerning its first relevance in insight moves was in amazing examination concerning assessing. In Great mid-1990s, scientcontinuets used beguilement hypo impressives proceed coordinated toward propose new esteeming systems for Internet administrator continuetration. Around Great same time, diversion impressive models were made for non-financial continuesues in frameworks, for instance, stream, attestation, in like way blockage control. Past amazing late 1990 s in like way mid-2000s, preoccupation hypo impressives proceed was winning associated coordinated 
toward remote frameworks. As eagerness for decentralized remote frameworks grew, so did amazing comfort concerning preoccupation impressiveoretic models coordinated toward concerning us upwards concerning continuance in the midst of understing such as mannering in like way anticipate Great execution concerning complex remote structures all together that can't win coordinated towardtally showed using cusdirected towardry streamlining gadgets. As radios get coordinated toward win arranged coordinated toward accomplish more advanced conformities, amazing suspicions concerning preoccupation impressive models turn indirected toward a still prevail match for future remote frameworks.

Entertainment hypo impressives proceed with, in light of current circumstances, multi-operadirected toward decision hypo impressives proceed with, continue playing sound, utility-boosting unique proceed with whose exercises impact each impresser's utilities. Redirection hypo impressives proceed has like continue gotten standard inventive capacity, in the midst of asscontinuance from Sylvia Nasr's life directed towardry concerning John Nash, A Prevail Mind (additionally amazing Hollywood film alteration concerning noteworthy book).Impressives days, even experts who are not eiimpressiver particularly included in the midst of redirection hypo impressives proceed are familiar in the midst of its essential thoughts, for instance, Great Nash amicability, in like way upwards concerning coordinated toward tests, for instance, amazing prisoners' difficulty. While Great minimum complex redirection impressive models expect all together that all rivals have complete information around one impressiveirhere's movement spaces in like way destinations, it proceed possible coordinated toward stretch out such models coordinated toward consider how lacking information impacts noteworthy result. Sadguyu, Prevail, in like way Ephremides apply Bayesian preoccupations coordinated toward Great continuesues concerning staying in remote frameworks.

\section{CONCLUSION}

Previously stated proceed with examination paper evaluates amazing execution concerning UMTS under EIGRP and RIP utilizing Gaming Impressiveory. Tear uses dynamic state controlling tradition in like way EIGRP relies on upon both component states coordination in like way interface state coordination. Both traditions contrast in estimations used for determination concerning wins course. Dcontinuetinctive traditions have moving pertinence unequivocal past keep setting execution concerning its parameters like num win concerning groups sent each second, wins concerning packages gotten each second in like way joining development for both RIP in like way EIGRP. Tear proceed less bewildering coordinated toward execute, on hardware and also programming in the midst of most noteworthy cerningiciency concerning time in like way memory however RIP can't win used for upwards concerning relevance as it puts aside more open door coordinated toward center in the midst of less adaptability as contemplated coordinated toward EIGRP. On exploring noteworthy eventual outcome concerning Great execution concerning dcontinuetinctive controlling conventions naming RIP in like way EIGRP over a condition for expense concerning transmcontinuesion, switch overhead in like way throughput we can say all together that EIGRP has wins execution all around as it has unimportant expense concerning transmcontinuesion, lesser switch overhead after RIP in like way most not amazing commendable throughput amongst all directing mythology.

\section{REFERENCES}

1. Jagdeep Singh, Rajiv Mahajan ,"Simulation Based comparitive study of RIP, OSPF and EIGRP, Vol3,Iss.8, pp.1-4, August 2013 ”, International Journal of Advanced Research in Computer Science and Software Engineering.

2. Rajendra kumar, Jitendra Vats, Arvind Kumar,"Comparitive Study of Routing Protocols, Vol.3, iss.6, pp.1-5,2011”, International Journal of Computer Science and Information Technology.

3. Vetriselvan.V, Mahendran.M , "Survey on RIP, OSPF and EIGRP Routing protocols", , Vol.5(2), iss.3, pp.2-7, 2014 International Journal of Computer Science and Information Technology.

4. Shah.A, Waqs J. Rana ,"Performance analysis of RIP and OSPF protocols in Network using OPNET", , Vol.10, Iss.6, pp. 2-5,November 2013 International Journal of Computer Science Issues.

5. A. Abu," Comparison study between IPV4 \& IPV6", ,Vol.10, No 2, pp.3-4,2012 International Journal of Computer Science Issues.

6. Dan Pei,Xiaoliang Zhao, LAn Wang "Detection of Invalid Routing Announcements in RIP Protocol" , , Volume 665-0-5622 2652-6/10,June 2010 International Journal of Computer Applications".

7. R. Coltun,et al., "RFC 5340 OSPF for IPv6,"IETF,vol.24, Iss 6,pp.352-365,2010.

8. IKram Ud Din,Saeed Mahfooz, Muhammad Adnan, "Analysis of the Routing Protocols in Real Time Transmission: A Comparitive Study", , Vol 4, Iss.5, pp.4- 6,2010 Global Journal of Computer Science and Technology.

9. Pankaj Rakheja, Prabhjot kaur, Anjali gupta, Aditi Sharma, "Performance Analysis of RIP, OSPF,IGRP and EIGRP Routing Protocols in a IEEE Sponsored 2nd International Conference on Innovations in Information Embedded and Communication Systems ICIIECS'15 Network", (0975-888)Volume 48-No.18, pp.210- 224,2012 International Journal of Computer Applications.

10. S. G. Thornier, "Dynamic Routing protocol implementation decision between EIGRP, OSPF and RIP based in technical background using OPNET Modeler", in Proc.Second Bangkok,Thailand,Pp191-195,2010 International Conference on Computer and Network Technology(ICCNT), 
11. B. Fortz and M. Thorup,(2002) "Optimizing OSPF/IS-IS weights in a changing world,",vol.20,no.4,pp.75-80,2002 IEEE Journal on Selected Areas in Communications.

12. www.safaribooksonline.com IEEE Sponsored 2nd International Conference on Innovations in Information Embedded and Communication Systems ICIIEC.

13. J. H. Schiller, "Mobile Communications", D.Kindersley, second edition, pp. 149-152.

14. Y. WANG, "QPPS: QoS Provision Packet Scheduling Algorithm in High Speed Downlink Packet Access", Wseas Transactions on communication Issue II, Volume 8, November 2009.

15. B. Walke, R. Seidenberg and M. P. Althoff, "UMTS: The Fundametals", John Wiley \& Sons, Ltd ISBN:0-470- 84557-0, 2003.

16. M. Chen, G. Liu, G. Zhu and X. Zhu, "A novel internetworking architecture between WLAN and UMTS cellular networks using RoF technique", , Shangai, China, pp. 693-697, May 2008 Proceedings of IEEE International Conference on Circuits and Systems for Communications.

17. P. Rakheja, P. Kaur, A. Gupta and A. Sharma, "Performance Analysis of RIP, OSPF, IGRP and EIGRP Routing Protocols in a Network" (0975 888) Volume 48- No.18, June 2012 International Journal of Computer Applications.

18. G.Jayakumar and G.Ganapathy, "Performance Comparison of Mobile Ad-hoc Network Routing Protocol", , VOL.7 No.1l, pp. 77-84, November 2007 IJCSNS International Journal of Computer Science and Network Security 